\title{
Commercial complementary food consumption is prospectively associated with added sugar intake in childhood
}

\author{
Kristina Foterek ${ }^{1}$, Anette E. Buyken ${ }^{1}$, Katja Bolzenius ${ }^{1}$, Annett Hilbig ${ }^{2}$, Ute Nöthlings ${ }^{1}$ and Ute Alexy ${ }^{1 *}$ \\ ${ }^{1}$ Nutritional Epidemiology, Department of Nutrition and Food Sciences, Dortmund Nutritional and Anthropometric \\ Longitudinally Designed (DONALD) Study, University of Bonn, 44225 Dortmund, Germany \\ ${ }^{2}$ Research Institute of Child Nutrition (FKE), University of Bonn, 44225 Dortmund, Germany \\ (Submitted 20 August 2015 - Final revision received 10 February 2016 - Accepted 10 March 2016 - First published online 15 April 2016)
}

\section{Abstract}

Given that commercial complementary food (CF) can contain high levels of added sugar, a high consumption may predispose to a preference for sweet taste later in life. This study examined cross-sectional associations between commercial CF consumption and added sugar intake in infancy as well as its prospective relation to added sugar intake in pre-school and primary-school age children. In all, 288 children of the Dortmund Nutritional and Anthropometric Longitudinally Designed Study with 3-d weighed dietary records at 0.5 and 0.75 (infancy), 3 and 4 (pre-school age) and 6 and 7 years of age (primary-school age) were included in this analysis. Individual commercial CF consumption as percentage of total commercial $\mathrm{CF}(\% \mathrm{cCF})$ was averaged at 0.5 and 0.75 years. Individual total added sugar intake (g/d, energy percentage/d) was averaged for all three age groups. Multivariable logistic and linear regression models were used to analyse associations between \%cCF and added sugar intake. In infancy, a higher \% $\mathrm{cCF}$ was associated with odds for high added sugar intake from $\mathrm{CF}$ and for high total added sugar intake ( $>75$ th percentile, $P<0.033$ ). Prospectively, a higher \%cCF was related to higher added sugar intake in both pre-school $(P<0.041)$ and primary-school age children $(P<0.039)$, although these associations were attenuated in models adjusting for added sugar intake in infancy. A higher \% CCF in infancy may predispose to higher added sugar intake in later childhood by virtue of its added sugar content. Therefore, offering home-made CF or carefully chosen commercial CF without added sugar might be one strategy to reduce sugar intake in infancy and later on.

\section{Key words: Infant feeding: Commercial complementary foods: Home-made complementary foods: Added sugar: Sweet} preference

High intakes of dietary sugar are extensively discussed to have adverse health consequences. Concerns relating to high sugar intakes comprise its contribution to the development of dental caries, excess weight gain, type 2 diabetes and CVD, as well as a lower nutritional adequacy of the diet, as added sugar itself does not provide essential nutrients ${ }^{(1-4)}$. Therefore, current dietary guidelines do emphasise the limitation of dietary sugar intake, focusing on the avoidance of sugar added during processing (added sugar) rather than sugars naturally occurring in foods ${ }^{(5)}$. More recently, the World Health Organization ${ }^{(4)}$ published new guidelines now setting a population goal $<10$ energy percentage (En\%) for free sugars (added sugars + sugars naturally present in fruit juices and fruit juice concentrates) and a conditional recommendation to further limit free sugar intake $<5 \mathrm{En} \%$ to achieve additional health benefits.

It is therefore of relevance to investigate factors shaping added sugar intake levels already in childhood. Although humans are born with a natural preference for sweet taste, which continues into childhood but then attenuates in adolescence and adulthood ${ }^{(6-8)}$, children's liking of sweet-tasting foods is not solely a consequence of their inborn taste preferences. Sensory properties of foods given early in life and repeated exposure to specific flavours can shape later taste preferences and food choice ${ }^{(6-8)}$. Apart from early exposure to a variety of flavours transmitted via amniotic fluid or breast milk ${ }^{(6,9)}$, flavour experiences from complementary food (CF) might also shape long-term dietary behaviour ${ }^{(10,11)}$. Added sugar content of CF may thus represent a relevant modifiable factor shaping long-term sweet preference.

Although there is a broad consensus in infant feeding recommendations that $\mathrm{CF}$ should contain preferably no or only a minimum level of added sugar or other sweeteners ${ }^{(12,13)}$, commercial $\mathrm{CF}$, that is, the main source of $\mathrm{CF}^{(14)}$, and commercial toddler foods can contain high levels of (added)

Abbreviations: \%cCF, percentage of commercial complementary food; CF, complementary food; DONALD Study, Dortmund Nutritional and Anthropometric Longitudinally Designed Study; En\%, energy percentage.

* Corresponding author: U. Alexy, fax +49 23171 1581, email alexy@uni-bonn.de 
sugar $^{(15-20)}$, although a detailed representative survey is lacking. This may entail the development of less-favourable taste and food preferences in contrast to a weaning diet concentrating on home-made $\mathrm{CF}$.

We thus hypothesised that a higher consumption of commercial CF and the concomitant early exposure to added sugar in infancy may predispose to higher intakes of added sugar in later childhood. To address this hypothesis, the cross-sectional associations between commercial CF consumption and total added sugar intake in infancy as well as the prospective relation to added sugar intake in pre-school and primary-school age were investigated.

\section{Methods}

\section{Study design and population}

The current analysis is based on data from the DOrtmund Nutritional and Anthropometric Longitudinally Designed (DONALD) Study, an ongoing open-cohort study. Details of the study design have been described previously ${ }^{(21)}$. In brief, as recruitment began in 1985, detailed information concerning diet, growth, development and metabolism has been collected in healthy subjects between infancy and early adulthood. Every year, approximately forty infants are newly recruited and first examined at the age of 3 months. Each child returns for three more visits in the 1st year, two in the 2nd year and then annually until young adulthood. The non-invasive assessments include 3-d weighed dietary records, interviews on lifestyle, anthropometric measurements and a medical examination. The study was approved by the Ethics Committee of the University of Bonn, and all examinations were performed with parental written consent and later on with the children's consent ${ }^{(21)}$.

The inclusion criterion for this analysis was the availability of three pairs of plausible 3 -d weighed dietary records at 0.5 and 0.75 (infancy), 3 and 4 (pre-school age), and 6 and 7 (primaryschool age) years of age. As the starting study sample included children and adolescents between the age of 2 and 18 years, data for the first few years of life were not always available ${ }^{(21)}$. In addition, many recruited children have not yet reached 7 years of age. Participants who were still fully breast- or formulafed at the age of 0.5 years were excluded from this analysis ( $n$ 46). Thus, 288 children born between 1985 and 2007 were included in the study sample.

\section{Nutritional assessment}

Dietary intake was assessed by using 3-d weighed dietary records. The parents of each child were instructed to weigh and record all foods and beverages consumed by their child using electronic food scales $( \pm 1 \mathrm{~g})$ on 3 consecutive days. The participants chose the day of the beginning of dietary recording within a given period of time. Moreover, foods and beverages consumed during any type of child care were recorded. Recipes (ingredients and preparation) of home-made CF as well as a detailed description including the type and brand of commercial $\mathrm{CF}$ were also requested. If exact weighing was not possible, semi-quantitative recording with household measures (e.g. number of glasses, cups and spoons) was allowed. A trained dietitian visited the family at home and checked the dietary records for accuracy and completeness.

Subsequently, any recorded food or drink was entered into the continuously updated in-house food composition database LEBTAB, which incorporates information from standard nutrient tables. For commercial food products, for example, commercial $\mathrm{CF}$, energy and nutrient contents were estimated using recipe simulation based on the labelled ingredients and nutrients. As the labelled ingredients are listed in decreasing order of their weight in the final product, they allow quantitative estimations of their amounts. A deviation lower than $\pm 5 \%$ of the food's calculated nutrient content from the labelled content was accepted. Any new food product that already exists in the database, but has changed in composition (e.g. new ingredients, fortification), received a new food code with a new simulation $^{(22)}$.

\section{Infant feeding}

CF was defined as all semi-solid, puréed or mashed foods fed using a spoon during infancy. Commercial CF was defined as all industrially processed, pre-packaged, CF foods (from jars or packets). Home-made CF was defined as all self-prepared, semi-solid, puréed or mashed foods made from scratch ${ }^{(10,14)}$. If home-made $\mathrm{CF}$ was consumed together with commercial CF products within a meal (e.g. home-made vegetable purée in combination with a commercial baby-meat jar), the individual ingredients were assigned to their respective preparation methods. Snack foods intended for infants and toddlers (e.g. biscuits or cereal bars) were not included in the definition of CF (exposure variable) because of the only minor differences in taste and texture between commercial and home-made products. For the purpose of this analysis, snack foods were considered as table foods, and thus still accounted for total energy intake as well as for total added sugar intake (outcome variable). Total $\mathrm{CF}$ consumption $(\mathrm{g} / \mathrm{d})$ was summed up for every record at 0.5 and 0.75 years of age. As we were interested in the habitual commercial CF consumption in infancy we then calculated average percentage of commercial $\mathrm{CF}$ (\%cCF) by dividing commercial CF (g/d) by total CF (g/d).

\section{Total added sugar intake and food sources of added sugar}

Added sugar is defined as sugars and syrups that are added to foods or beverages during processing or preparation at home and/or by the food industry ${ }^{(23)}$. The following foods were defined as added sugar: white sugar, brown sugar, raw sugar, maize syrup, maize syrup solids, high-fructose maize syrup, malt syrup, maple syrup, pancake syrup, fruit syrup, fructose sweetener, liquid fructose, honey, molasses, anhydrous dextrose and crystal dextrose ${ }^{(24)}$. Conversely, naturally occurring sugars such as lactose in milk/formula or fructose in fruits were not included. The sugar content of maltodextrin was defined as $50 \%$ of its carbohydrate content, because maltodextrin consists of a mixture of monomers, dimers, oligomers and polymers of glucose. Sugar substitutes and added sugar in medicines were neglected $^{(24)}$. 
In this analysis, different food sources of added sugar were also examined: added sugar from commercial, home-made and total CF (definitions see above), added sugar from beverages (defined as added sugar from regular soft drinks, diet soft drinks and fruit drinks), added sugar from sweets (defined as added sugar from candy, chocolate, sweet spreads, ice cream, pastries and other sweeteners), added sugar from milk products (defined as added sugar from infant formula other than lactose, milk and milk products), and added sugar from other food sources (i.e. the remainder of food sources, e.g. breakfast cereals, convenience products).

Individual data on daily absolute $(\mathrm{g} / \mathrm{d})$ and relative added sugar intakes $(\mathrm{En} \% / \mathrm{d})$ as well as energy intakes $(\mathrm{kJ} / \mathrm{d})$ were derived from the mean of the $3 \mathrm{~d}$ of recording. For each participant, dietary intake values from 0.5 and $0.75,3$ and 4,6 and 7 years of age were averaged to obtain estimates of the habitual intake in infancy, pre-school and primary-school age, respectively.

\section{Potentially confounding factors}

Parents were interviewed about family characteristics (e.g. parental education) as well as weighed and measured by the study staff on their child's admission to the study, and thereafter at 4-year intervals. Information on the child's birth characteristics was abstracted from the 'Mutterpass', a standardised document given to all pregnant women in Germany.

In addition to the dietary records, breast-feeding status was enquired by the study dietitians at each of the first visits until the infant was fully weaned. Ongoing breast-feeding was defined as any breast-feeding (full or partial) at 3 months and partial breast-feeding at 6 months of age (full breast-feeding at 6 months of age was an exclusion criterion).

For this analysis, the following characteristics were considered as potential confounders: total and remaining energy intake (i.e. total energy intake minus energy intake from added sugar, $\mathrm{kJ} / \mathrm{d}$ ) of the child in the respective age group, ongoing breastfeeding at 3 and 6 months of age (yes/no, respectively), date of birth, birth weight $(\mathrm{g})$, being firstborn (yes/no), high maternal educational status ( $\geq 12$ years of schooling, yes/no), maternal age at birth (years) and maternal overweight (BMI $\geq 25 \mathrm{~kg} / \mathrm{m}^{2}$, yes/no). For missing values, the respective median of the total sample ( $n 3$ for maternal overweight, median: $23.4 \mathrm{~kg} / \mathrm{m}^{2}$ ) was used.

\section{Statistical analyses}

$\mathrm{SAS}^{\circledR}$ procedures (version 9.1.3; Statistical Analysis System) were used for all data analyses. $P$ values $<0.05$ were considered as statistically significant.

For all the analyses, the predictor variable \%cCF was categorised into tertiles (low $\leq 43.0 \%$, medium $43.1-\leq 71.0 \%$, high $>71.0 \%$ ). Owing to the number of zero values and the resulting skewness of the outcome variables (total added sugar intake and added sugar intake from $\mathrm{CF}$ in infancy), multivariable logistic regression models (PROC LOGISTIC in SAS) were used to calculate OR for associations between \% $\mathrm{cCF}$ and high added sugar intake in infancy. The outcome variables total added sugar intake and added sugar intake from CF were defined as high, if intake values exceeded the 75th percentile (high total added sugar intake $>4.6 \mathrm{En} \% / \mathrm{d}$ or $8.1 \mathrm{~g} / \mathrm{d}$, high added sugar intake from $\mathrm{CF}>2.6 \mathrm{En} \% / \mathrm{d}$ or $4.4 \mathrm{~g} / \mathrm{d}$, respectively). The outcome variables total added sugar intakes in pre-school and primary-school age were log-transformed to obtain normal distribution. Multivariable linear regression models (PROC GLM in SAS) were used to analyse the prospective associations between \%cCF in infancy and added sugar intake (g/d, En\%/d) in pre-school and primary-school age.

As analyses indicated no interactions of sex with the associations between \%cCF in infancy and added sugar intake (total or from CF), data from girls and boys were pooled for all further analyses. Results are presented as adjusted means in tertiles of $\%$ CCF in infancy.

All basic models were adjusted for sex and date of birth. For the adjusted models, each potential confounder was initially considered separately and only those variables that (a) had a significant, independent effect on the outcome variable, (b) modified regression coefficients $(\beta)$ in the basic models by $\geq 5 \%$ or (c) led to an improvement of the coefficient of determination $\left(R^{2}\right)$ by $\geq 10 \%{ }^{(25)}$ were considered in the final adjusted models.

In a further step, we additionally adjusted for absolute added sugar intake levels in infancy, so as to investigate whether added sugar intake in infancy may mediate any potential association between \%cCF and later added sugar intake.

\section{Results}

\section{Study sample characteristics}

Descriptive data of the study sample are presented in Table 1. The analysis included 134 girls (46.5\%) and 154 boys (53.5\%). Two-thirds of the participants were fully breast-fed at 3 months of age; more than half of them were still partially breast-fed at 6 months of age. Nearly $65 \%$ were the firstborn in their family. Mean maternal age at birth was 32.6 years, with $<30 \%$ of the mothers being overweight and almost $70 \%$ having a high educational level.

The median \%cCF in infancy ( 0.5 and 0.75 years) was $57.7 \%$. In all, fourteen participants received no commercial CF in both records at all, whereas eight of them consumed commercial CF only (data not shown). As both extremes reflect common dietary practices, these participants were retained in the analysis

\section{Commercial complementary food, added sugar intake and food sources of added sugar}

In total, thirty-three infants (11.5\%) did not consume any added sugar in infancy (data not shown). Table 2 illustrates that $<2 \mathrm{~g}$ of total added sugar intake in infancy came from $\mathrm{CF}$, with higher added sugar intake levels from commercial than home-made CF $(P=0.012)$. Median total added sugar intake increased from $2 \cdot 3$ En\%/d in infancy to 11.9 and 13.4 En\% in pre-school and primary-school age, respectively $(P<0.0001)$. In both preschool and primary-school age, median added sugar intakes (En\%) exceeded the current World Health Organization 
recommendation ${ }^{(4)}$. In infancy, the majority of added sugar came from total CF, whereas more than half of the infants did not receive any added sugar from the other food sources. Conversely, sweets were the main source of added sugar in both pre-school and primary-school age, followed by milk products.

\section{Relation of infant commercial complementary food consumption to added sugar intake}

In infancy, \%cCF was significantly associated with total added sugar intake as well as added sugar intake from CF for both relative (En\%) and absolute added sugar intakes $(\mathrm{g} / \mathrm{d})$. Infants with a high commercial CF consumption had higher odds for high consumption of added sugar from CF as well as for a high total added sugar intake (Table 3). Given the generally low total added sugar intakes in infancy, the absolute differences in sugar intake between tertiles of commercial CF consumption are less distinct.

Table 1. Characteristics of the analysed Dortmund Nutritional and Anthropometric Longitudinally Designed Study sample, born in 1985-2007 (Numbers and percentages; mean values and standard deviations)

\begin{tabular}{|c|c|c|}
\hline \multirow[b]{2}{*}{ Variables } & \multicolumn{2}{|c|}{ Values } \\
\hline & $n$ & $\%$ \\
\hline \multicolumn{3}{|l|}{ Early-life characteristics } \\
\hline \multicolumn{3}{|l|}{ Birth weight (g) } \\
\hline Mean & \multirow{2}{*}{\multicolumn{2}{|c|}{$\begin{array}{r}3456 \\
48 ?\end{array}$}} \\
\hline SD & & \\
\hline Full breast-feeding at 3 months & 193 & $67 \cdot 0$ \\
\hline Partial breast-feeding at 3 months & 24 & 8.3 \\
\hline Partial breast-feeding at 6 months & 163 & $56 \cdot 6$ \\
\hline \multicolumn{3}{|l|}{ Family characteristics } \\
\hline Firstborn & 186 & $64 \cdot 6$ \\
\hline \multicolumn{3}{|l|}{ Maternal age at birth (years) } \\
\hline Mean & \multirow{2}{*}{\multicolumn{2}{|c|}{$\begin{array}{r}32.6 \\
3.6\end{array}$}} \\
\hline SD & & \\
\hline Maternal overweight* & 82 & 28.5 \\
\hline High maternal educational status $\dagger$ & 201 & $69 \cdot 8$ \\
\hline
\end{tabular}

${ }^{*} \mathrm{BMI} \geq 25 \mathrm{~kg} / \mathrm{m}^{2}$.

$\dagger \geq 12$ years of schooling
In prospective analyses, higher \%cCF in infancy was associated with higher relative and absolute added sugar intakes in both pre-school and primary-school age (Table 4). In adjusted models, pre-schoolers in the highest tertile of infant \%cCF had a $10 \%$ higher relative added sugar intake than those in the lowest tertile. In primary-school age, relative added sugar intake was $12 \%$ higher in the highest as compared with the lowest tertile of infant \%cCF. When the final models were further adjusted for absolute added sugar intake in infancy, these prospective associations were attenuated and most of them were no longer significant. The attenuation was even more pronounced when models for primary-school age were adjusted for absolute added sugar intake in pre-school age (data not shown).

\section{Discussion}

The present study provides novel evidence of a sustained relationship between a high contribution of commercial CF in infancy and higher total added sugar intakes throughout childhood. A higher consumption of commercial CF in infancy entailed a higher added sugar intake already during infancy and was also prospectively related to higher added sugar intakes in pre-school and primary-school age. Importantly, the prospective associations were partly explained by total added sugar intake levels in infancy, indicating that higher amounts of added sugar in commercial $\mathrm{CF}^{(15,18,26)}$ may be responsible for this adverse relationship.

For the current German baby-food market, the online CF database 'Babynahrungsproduktsuche' illustrates that $17 \%$ of all spoonable CF products contain added sugar ${ }^{(18)}$. However, information on exact amounts of added sugar is not given in the database. A recent evaluation of ours on the nutrient composition of home-made and commercial CF revealed that added sugar was present in nearly a quarter of all CF meals consumed in the DONALD Study, with the highest contents in commercial dairy-fruit meals (third quartile: $7 \mathrm{~g} / 100 \mathrm{~g}$ ). In cereal-milk meals as well as in cereal-fruit meals, added sugar content was significantly higher in commercial than in home-made meals ${ }^{(26)}$.

Table 2. Total added sugar intake and relative contribution of food sources to added sugar intake of the analysed Dortmund Nutritional and Anthropometric Longitudinally Designed Study sample in infancy, pre-school age and primary-school age*

(Medians and interquartile ranges (IQR, Q1-Q3))

\begin{tabular}{|c|c|c|c|c|c|c|}
\hline & \multicolumn{2}{|c|}{ Infancy $(0.5-0.75$ years $)$} & \multicolumn{2}{|c|}{ Pre-school age (3-4 years) } & \multicolumn{2}{|c|}{ Primary-school age (6-7 years) } \\
\hline & Median & IQR & Median & IQR & Median & IQR \\
\hline \multicolumn{7}{|c|}{ Total added sugar intake } \\
\hline $\mathrm{g} / \mathrm{d}$ & 4.0 & $0.7-8 \cdot 1$ & $34 \cdot 2$ & $26 \cdot 9-42 \cdot 6$ & $49 \cdot 7$ & $38.9-63.2$ \\
\hline $\mathrm{En} \% / \mathrm{d}$ & $2 \cdot 3$ & $0.5-4 \cdot 6$ & 11.9 & $9 \cdot 4-14.7$ & 13.4 & $10 \cdot 9-16 \cdot 7$ \\
\hline \multicolumn{7}{|c|}{ Added sugar intake from different food sources $(\mathrm{g} / \mathrm{d})$} \\
\hline $\mathrm{CF}$ & 1.8 & $0.3-4.4$ & & & & \\
\hline Commercial CF & 0.6 & $0.0-2 \cdot 8$ & & & & \\
\hline Home-made CF & 0.1 & $0.0-1.4$ & & & & \\
\hline Beverages & 0.0 & $0.0-0.0$ & 1.8 & $0.0-6 \cdot 0$ & $4 \cdot 0$ & $0.5-9.4$ \\
\hline Milk products & 0.0 & $0.0-2 \cdot 3$ & $6 \cdot 6$ & $3 \cdot 6-11 \cdot 1$ & $8 \cdot 0$ & $5 \cdot 0-13 \cdot 1$ \\
\hline Sweets & 0.0 & $0.0-0.4$ & 18.9 & $13 \cdot 7-25 \cdot 4$ & $26 \cdot 6$ & $21 \cdot 3-34 \cdot 6$ \\
\hline Other foods & 0.0 & $0.0-0.0$ & $2 \cdot 3$ & $1 \cdot 0-5 \cdot 0$ & 4.8 & $2 \cdot 6-8 \cdot 1$ \\
\hline
\end{tabular}

En\%, energy percentage; $\mathrm{CF}$, complementary food.

* Added sugar intake levels $(\mathrm{g} / \mathrm{d}, \mathrm{En} \% / \mathrm{d})$ were derived from the mean of both 3-d weighed dietary records for the respective age group. 
Table 3. Cross-sectional relation between percentage of commercial complementary food (\%cCF) in infancy and high total added sugar intake or high added sugar intake from complementary food (CF) in infancy (basic and adjusted multivariable logistic regression models) of the analysed Dortmund Nutritional and Anthropometric Longitudinally Designed Study sample*

(Medians and interquartile ranges (IQR); odds ratios, adjusted odds ratios and $95 \%$ confidence intervals)

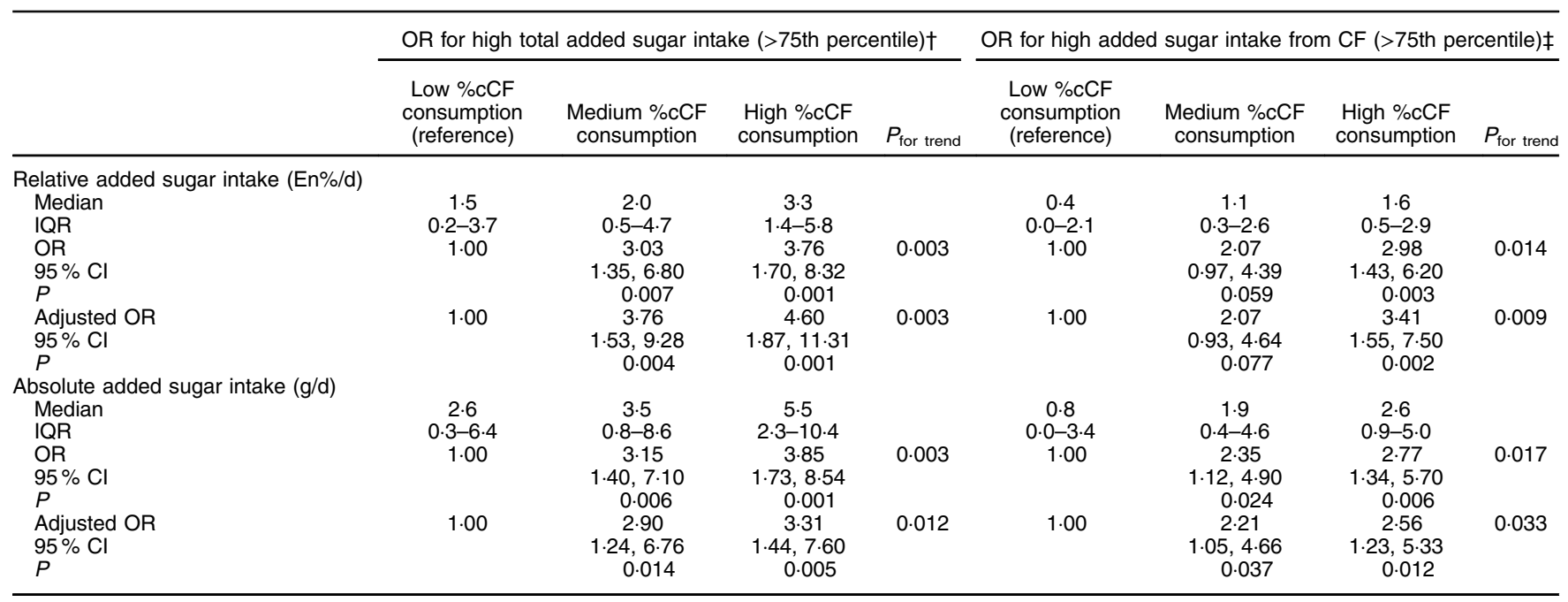

En\%, energy percentage.

* Significant differences between tertiles of \%cCF consumption (low, medium, high \%cCF) were tested using multivariable logistic regression for ordinal dependent variables. Basic models: adjusted for sex and date of birth. Adjusted models: basic model +adjustment for ongoing breast-feeding at 3 and 6 months (yes/no, respectively) in the model for absolute total added sugar intake and additionally for total energy intake (kJ/d) in the model for relative total added sugar intake; basic model + adjustment for ongoing breastfeeding at 6 months (yes/no) in the model for absolute added sugar intake from CF and additionally for total energy intake ( $\mathrm{kJ} / \mathrm{d})$ and maternal overweight (BMI $\geq 25 \mathrm{~kg} / \mathrm{m}^{2}$, yes/no) in the model for relative added sugar intake from CF.

$\dagger$ High total added sugar intake $>4.6 \mathrm{En} \% / \mathrm{d}$ and $8.1 \mathrm{~g} / \mathrm{d}$, respectively.

$\ddagger$ High added sugar intake from CF $>2.6 \mathrm{En} \% / \mathrm{d}$ and $4.4 \mathrm{~g} / \mathrm{d}$, respectively.

Table 4. Prospective associations between percentage of commercial complementary food (\%cCF) in infancy and added sugar intake ( $\mathrm{g} / \mathrm{d}$ and energy percentage $(\mathrm{En} \%) / \mathrm{d})$ in pre-school and primary-school age (basic and adjusted regression models) of the analysed Dortmund Nutritional and Anthropometric Longitudinally Designed Study sample*

(Adjusted mean values and $95 \%$ confidence intervals)

\begin{tabular}{|c|c|c|c|c|c|c|c|c|c|c|c|c|c|c|}
\hline & \multicolumn{7}{|c|}{ Pre-school age (3-4 years) } & \multicolumn{7}{|c|}{ Primary-school age (6-7 years) } \\
\hline & \multicolumn{2}{|c|}{$\begin{array}{l}\text { Low \%cCF } \\
\text { consumption }\end{array}$} & \multicolumn{2}{|c|}{$\begin{array}{l}\text { Medium \%cCF } \\
\text { consumption }\end{array}$} & \multicolumn{2}{|c|}{$\begin{array}{l}\text { High \%cCF } \\
\text { consumption }\end{array}$} & \multirow[b]{2}{*}{$P_{\text {for trend }} \dagger$} & \multicolumn{2}{|c|}{$\begin{array}{l}\text { Low \%cCF } \\
\text { consumption }\end{array}$} & \multicolumn{2}{|c|}{$\begin{array}{l}\text { Medium \%cCF } \\
\text { consumption }\end{array}$} & \multicolumn{2}{|c|}{$\begin{array}{l}\text { High \%cCF } \\
\text { consumption }\end{array}$} & \multirow[b]{2}{*}{$P_{\text {for trend }} t$} \\
\hline & Mean & $95 \% \mathrm{Cl}$ & Mean & $95 \% \mathrm{Cl}$ & Mean & $95 \% \mathrm{Cl}$ & & Mean & $95 \% \mathrm{Cl}$ & Mean & $95 \% \mathrm{Cl}$ & Mean & $95 \% \mathrm{Cl}$ & \\
\hline \multicolumn{15}{|c|}{ Relative added sugar intake $(\mathrm{En} \% / \mathrm{d})$} \\
\hline Basic model & 10.9 & $10 \cdot 2$ & 11.6 & $10 \cdot 8,12.5$ & $12 \cdot 4$ & $11 \cdot 5,13 \cdot 3$ & 0.023 & 12.5 & $11 \cdot 7,13 \cdot 3$ & $13 \cdot 2$ & $12 \cdot 4,14 \cdot 2$ & 14.2 & $13 \cdot 2,15 \cdot 1$ & 0.013 \\
\hline Adjus & 11.3 & 10.5, & $12 \cdot 4$ & 11.4, & $12 \cdot 7$ & & & $12 \cdot 6$ & & 13.5 & & $14 \cdot 3$ & & 0.017 \\
\hline $\begin{array}{l}\text { Adjusted model + added } \\
\text { sugar intake }(\mathrm{g} / \mathrm{d}) \text { in infancy }\end{array}$ & 11.4 & $10 \cdot 6,12 \cdot 3$ & $12 \cdot 3$ & $11 \cdot 4,13 \cdot 3$ & $12 \cdot 6$ & $11 \cdot 7,13 \cdot 6$ & 0.116 & $12 \cdot 7$ & $11 \cdot 8,13 \cdot 6$ & $13 \cdot 4$ & $12.5,14.4$ & $14 \cdot 2$ & $13 \cdot 3,15 \cdot 2$ & 0.045 \\
\hline \multicolumn{15}{|c|}{ Absolute added sugar intake ( $\mathrm{g} / \mathrm{d})$} \\
\hline Basic model & & $28.5,33.4$ & 34.0 & $31 \cdot 4,36 \cdot 8$ & $35 \cdot 3$ & $32 \cdot 6,38 \cdot 2$ & 0.021 & 45.9 & $42 \cdot 5,49.7$ & 48.0 & $44 \cdot 5,52 \cdot 1$ & $50 \cdot 9$ & $47 \cdot 1,55 \cdot 0$ & 0.054 \\
\hline Adjusted model & 32.0 & $29.5,34.7$ & $36 \cdot 4$ & $33.4,39.6$ & 36.4 & $33.7,39.4$ & 0.035 & 45.7 & $41.9,49.8$ & 48.9 & $44.9,53 \cdot 3$ & 51.4 & $47 \cdot 4,55 \cdot 7$ & 0.039 \\
\hline $\begin{array}{l}\text { Adjusted model + added } \\
\text { sugar intake }(\mathrm{g} / \mathrm{d}) \text { in infancy }\end{array}$ & 32.4 & $29 \cdot 8,35 \cdot 1$ & $36 \cdot 2$ & $33 \cdot 2,39 \cdot 4$ & $36 \cdot 0$ & $33 \cdot 3,39 \cdot 0$ & 0.116 & $46 \cdot 8$ & $43 \cdot 1,50 \cdot 7$ & 49.4 & $45 \cdot 5,53 \cdot 6$ & 51.5 & $47 \cdot 6,55 \cdot 7$ & 0.106 \\
\hline
\end{tabular}

* Basic models: adjusted for sex and date of birth. Adjusted models: basic model + adjustment for being firstborn (yes/no) and high maternal educational status ( $\geq 12$ years of schooling, yes/no) in the pre-school age models; basic model + adjustment for high maternal educational status ( $\geq 12$ years of schooling, yes/no) and total energy intake at 6-7 years ( $\mathrm{kJ} / \mathrm{d}$ ) in primary-school age models (relative added sugar intake); basic model+adjustment for high maternal educational status ( $\geq 12$ years of schooling, yes $/ \mathrm{no}$ ), ongoing breast-feeding at 3 months (yes $/ \mathrm{no}$ ), and remaining energy intake at 6-7 years (kJ/d) in primary-school age models (absolute added sugar intake).

$\dagger P_{\text {for trend }}$ refers to $P$ values obtained in linear regression models.

A high commercial CF intake in infants might also act as a proxy for a less-favourable infant diet with other sweetened products apart from $\mathrm{CF}$, such as sugar-based instant teas, baby rusks/cookies or sweetened follow-on formula, as supported by the findings of a pattern analysis from the $\mathrm{UK}^{(27)}$. Therefore, it may not only be the added sugar contained in commercial CF itself but also the added sugar from all remaining foods and drinks consumed by infants, with a high commercial CF consumption resulting in a high added sugar intake in later childhood.

In our study, the association between a high \%cCF and a higher total added sugar intake also persisted in the long term. It is well known from experimental studies that the inborn preference for sweet taste is further enhanced by the early intake of and repeated exposure to sugar and sweet foods ${ }^{(7)}$. Infants fed sweetened water after birth showed a greater preference for 
sweetened water at 6 months, 2 years and 6 years of age compared with those fed plain water ${ }^{(28-30)}$. In addition, the time of introduction of sweetened foods may affect children's added sugar intake: in a Swedish study, frequent eaters of sweets at 1 year of age had been introduced to sweets nearly 1 month earlier than the other infants ${ }^{(31)}$

The effect of repeated exposure shaping sweet taste preferences is not only restricted to early infancy but has also been shown during childhood. Children (4-7 years old) whose mothers reported to routinely add sugar to their child's diet were shown to prefer apple juices and cereals with a higher added sugar content compared with children without added sugar experience ${ }^{(32)}$. Further, even an 8-d short-term exposure to sweetened orangeade in 6-11-year-old children increased the preference for this orangeade after the intervention. This effect was also shown for an overall preference of high sugar concentration independent of the food that sucrose was added to ${ }^{(33)}$. Although children do not have full autonomy in making their food choices, their actual food intake results from the interaction between the parental provision of foods and the child's individual taste preferences. Accordingly, our findings suggest that the habitual intake of (sweetened) commercial CF leads to a general higher preference for sweetened foods later in childhood.

To the best of our knowledge, our study is the first to describe a prospective association between commercial $\mathrm{CF}$ consumption and added sugar intake, although we cannot prove causality given the observational design. It could be argued that differences in sugar intake levels of $1-5 \mathrm{~g} / \mathrm{d}$ as reported in this study are lacking clinical relevance, yet our study suggests a potential and sustained relevance even of these relatively low total added sugar intake levels for later sugar intake. The association attenuated in the statistical analysis adjusting for total added sugar intake in infancy (and even more for total added sugar in pre-school age), indicating that a high early added sugar intake mediates this association - that is, early added sugar intake is an important determinant of added sugar intake in later childhood. As other studies demonstrated that sweetened snacks and beverages track between infancy and toddlerhood as well as pre-school age ${ }^{(34-37)}$, the consistency of the observed associations may reflect the tracking of early dietary patterns. Thus, our results underpin the proposed hypothesis that the consumption of sweetened foods and beverages later in life is facilitated by a high and frequent added sugar intake via commercial CF in early infancy.

A frequent intake of foods high in added sugar tends to be linked to an overall less-favourable diet in children ${ }^{(31,38-40)}$. From a public health perspective, our data suggest that providing CF without added sugar may therefore help protect children from excess added sugar intake later in childhood by not further enhancing their inborn sweet preference. Although it seems to be possible to achieve a reasonable diet quality and adequate nutrient intake even within high ranges of added sugar intake ${ }^{(41-43)}$, sugar addition in foods for children is not necessary to enhance their palatability and should be avoided. In an experimental study by Bouhlal et al. ${ }^{(44)}$, the level of added sugar did not affect the intake of fruit purée in 2-3-year-old toddlers ${ }^{(44)}$. Instead, repeated exposure increased the preference for an unknown food regardless of whether sugar was added or not ${ }^{(45)}$. Therefore, infant feeding guidelines should encourage parents to (at least occasionally) offer homemade CF or to choose commercial CF products without added sugar. The recent decrease in infants' added sugar intake during the last decade already indicates a desirable trend ${ }^{(46)}$.

Major strengths of our analysis include the detailed dietary assessment and the comprehensive food composition database allowing the differentiation between home-made and commercial CF. As added sugar content cannot be analytically determined $^{(5)}$, we were able to identify and calculate the amount of added sugar in composite commercial products as well as home-made recipes, as these could be broken down into their single ingredients on the basis of the in-house recipe simulation procedure ${ }^{(22)}$. Furthermore, information on several possible confounders such as early-life and parental characteristics was available.

However, the generalisability of our results may be limited, as the DONALD population is characterised by a rather high socioeconomic status $^{(21)}$. Several studies reported a high parental educational level being associated with a less-frequent intake of sweets and added sugar ${ }^{(31,47)}$ or an overall higher diet quality in their children ${ }^{(48,49)}$. Furthermore, added sugar intake may be underestimated in the current observational analysis as the under-reporting of sugar-rich foods is a common problem in nutritional surveys ${ }^{(50)}$. Therefore, the average total added sugar intake may be higher in the general population and with that the true associations even more distinct.

An additional limitation of our study is the lack of parental dietary data. Neither parents' added sugar intake nor their overall diet quality was assessed, and could therefore not been taken into account, although it is known to be a strong influencing factor in early infant nutrition.

\section{Conclusion}

The data from the DONALD Study suggest that the choice of home-made or commercial CF influences the added sugar intake from $\mathrm{CF}$ as well as the total intake of added sugar in infancy. A high commercial CF consumption in infancy may predispose to a higher added sugar intake in later childhood by virtue of its added sugar content. Therefore, offering home-made CF or carefully chosen commercial CF without added sugar might be one strategy to reduce added sugar intake in infancy and later in childhood. To strengthen these study findings, our first explorative results need to be confirmed in large-scale cohort studies. If associations are repeatedly found, well-planned intervention studies are required to allow assertions on causal inference.

\section{Acknowledgements}

The participation of all children and their families in the DONALD Study is gratefully acknowledged. The authors also thank the DONALD staff for carrying out the anthropometric measurements, for administering the questionnaires and for collecting and coding the dietary records. 
The DONALD Study is supported by the Ministry of Science and Research of North Rhine Westphalia, Germany.

The authors' contributions were as follows - A. E. B. and U. A. supervised the study; K. F. and U. A. designed the research; K. F. performed the statistical analysis and drafted the manuscript; A. E. B., K. B., A. H., U. N. and U. A. provided critical input on data analyses and early versions of the manuscript; U. A. had primary responsibility for the final content. All the authors read and approved the final version of the manuscript.

The authors have no financial or personal conflicts of interest to declare.

\section{References}

1. Alles MS, Eussen SR \& van der Beek EM (2014) Nutritional challenges and opportunities during the weaning period and in young childhood. Ann Nutr Metab 64, 284-293.

2. Moynihan PJ \& Kelly SAM (2014) Effect on caries of restricting sugars intake: systematic review to inform WHO guidelines. J Dent Res 93, 8-18.

3. Te Morenga L, Mallard S \& Mann J (2013) Dietary sugars and body weight: systematic review and meta-analyses of randomised controlled trials and cohort studies. BMJ 346, e7492.

4. World Health Organization (2015) Guideline: Sugars Intake for Adults and Children. Geneva: WHO. http://apps.who.int/ iris/bitstream/10665/149782/1/9789241549028_eng.pdf (accessed March 2015).

5. Hess J, Latulippe ME, Ayoob K, et al. (2012) The confusing world of dietary sugars: definitions, intakes, food sources and international dietary recommendations. Food Funct 3, 477-486.

6. Ventura AK \& Worobey J (2013) Early influences on the development of food preferences. Curr Biol 23, R401-R408.

7. Ventura AK \& Mennella JA (2011) Innate and learned preferences for sweet taste during childhood. Curr Opin Clin Nutr Metab Care 14, 379-384.

8. Mennella JA (2014) Ontogeny of taste preferences: basic biology and implications for health. Am J Clin Nutr 99, S704-S711.

9. Mennella JA \& Trabulsi JC (2012) Complementary foods and flavor experiences: setting the foundation. Ann Nutr Metab 60, Suppl. 2, 40-50.

10. Foterek K, Hilbig A \& Alexy U (2015) Associations between commercial complementary food consumption and fruit and vegetable intake in children - results of the DONALD study. Appetite 85, 84-90.

11. Coulthard H, Harris G \& Emmett P (2010) Long-term consequences of early fruit and vegetable feeding practices in the United Kingdom. Public Health Nutr 13, 2044-2051.

12. Koletzko BV, Bauer CP, Brönstrup A, et al. (2013) Säuglingsernährung und Ernährung der stillenden Mutter. Aktualisierte Handlungsempfehlungen des Netzwerk Gesund ins Leben Netzwerk Junge Familie, ein Projekt von IN FORM (Infant nutrition and nutrition for breastfeeding mothers. Updated practice recommendations of the network 'Healthy Start Young Family Network' a project of IN FORM). Monatsschr Kinderb 137, 1309-1314 (in German).

13. Agostoni C, Decsi T, Fewtrell M, et al. (2008) Complementary feeding: a commentary by the ESPGHAN committee on nutrition. J Pediatr Gastroenterol Nutr 46, 99-110.

14. Foterek K, Hilbig A \& Alexy U (2014) Breast-feeding and weaning practices in the DONALD study: age and time trends. J Pediatr Gastroenterol Nutr 58, 361-367.

15. Düren M \& Kersting M (2003) Das Angebot an Kinderlebensmitteln in Deutschland. Produktübersicht und ernährungsphysiologische Wirkung (The range of children's food products in Germany - product overview and nutritional valuation). Ernaehrungs Umschau 50, 16-21 (in German).

16. Elliott CD (2011) Sweet and salty: nutritional content and analysis of baby and toddler foods. J Public Health (Oxf) 33, 63-70.

17. Elliott CD \& Conlon MJ (2015) Packaged baby and toddler foods: questions of sugar and sodium. Pediatr Obes 10, 149-155.

18. Forschungsinstitut für Kinderernährung (2015) BeikostDatenbank 'Babynahrungsproduktsuche'. Informationssystem für die Bereiche Säuglings- und Kleinkindernährung (Complementary Food Database 'Babynahrungsproduktsuche' information system for infant and toddler nutrition) (in German). https://verbraucherfenster.hessen.de/service/ babynahrung-produktsuche (accessed January 2015).

19. Cogswell ME, Gunn JP, Yuan K, et al. (2015) Sodium and sugar in complementary infant and toddler foods sold in the United States. Pediatrics 135, 416-423.

20. Samuel L, Ethan D, Basch CH, et al. (2014) A comparative study of the sodium content and calories from sugar in toddler foods sold in low- and high-income New York City supermarkets. Glob J Health Sci $\mathbf{6}, 22-29$.

21. Kroke A, Manz F, Kersting M, et al. (2004) The DONALD study: history, current status and future perspectives. Eur J Nutr 43, 45-54.

22. Sichert-Hellert W, Kersting M, Chahda C, et al. (2007) German food composition database for dietary evaluations in children and adolescents. J Food Compos Anal 20, 63-70.

23. Institute of Medicine of the National Academies (2005) Dietary Reference Intakes: Energy, Carbohydrate, Fiber, Fat, Fatty Acids, Cholesterol, Protein, and Amino Acids. Washington, DC: Institute of Medicine of the National Academies. www. nal.usda.gov/fnic/DRI/DRI_Energy/energy_full_report.pdf (accessed June 2014).

24. Herbst A, Diethelm K, Cheng G, et al. (2011) Direction of associations between added sugar intake in early childhood and body mass index at age 7 years may depend on intake levels. J Nutr 141, 1348-1354.

25. Maldonado G \& Greenland S (1993) Simulation study of confounder-selection strategies. Am J Epidemiol 138, 923-936.

26. Hilbig A, Foterek K, Kersting M, et al. (2015) Home-made and commercial complementary meals in German infants: results of the DONALD study. J Hum Nutr Diet 28, 613-622.

27. Brazionis L, Golley RK, Mittinty MN, et al. (2012) Characterization of transition diets spanning infancy and toddlerhood: a novel, multiple-time-point application of principal components analysis. Am J Clin Nutr 95, 1200-1208.

28. Beauchamp GK \& Moran M (1982) Dietary experience and sweet taste preference in human infants. Appetite 3, 139-152.

29. Beauchamp GK \& Moran M (1984) Acceptance of sweet and salty tastes in 2-year-old children. Appetite 5, 291-305.

30. Pepino MY \& Mennella JA (2005) Factors contributing to individual differences in sucrose preference. Chem Senses 30 , Suppl. 1, 319-320.

31. Brekke HK, van Odijk J \& Ludvigsson J (2007) Predictors and dietary consequences of frequent intake of high-sugar, low-nutrient foods in 1-year-old children participating in the ABIS study. Br J Nutr 97, 176-181.

32. Liem DG \& Mennella JA (2002) Sweet and sour preferences during childhood: role of early experiences. Dev Psychobiol 41, 388-395.

33. Liem DG \& de Graaf C (2004) Sweet and sour preferences in young children and adults: role of repeated exposure. Physiol Behav 83, 421-429. 
34. Lioret S, McNaughton SA, Spence AC, et al. (2013) Tracking of dietary intakes in early childhood: the Melbourne InFANT program. Eur J Clin Nutr 67, 275-281.

35. Bjelland M, Brantsæter AL, Haugen M, et al. (2013) Changes and tracking of fruit, vegetables and sugar-sweetened beverages intake from 18 months to 7 years in the Norwegian Mother and Child Cohort Study. BMC Public Health 13, 793.

36. Park S, Pan L, Sherry B, et al. (2014) The association of sugarsweetened beverage intake during infancy with sugarsweetened beverage intake at 6 years of age. Pediatrics 134, Suppl. 1, S56-S62.

37. Lioret S, Betoko A, Forhan A, et al. (2015) Dietary patterns track from infancy to preschool age: cross-sectional and longitudinal perspectives. $J$ Nutr 145, 775-782.

38. Forshee RA \& Storey ML (2001) The role of added sugars in the diet quality of children and adolescents. J Am Coll Nutr 20, 32-43.

39. Livingstone MBE \& Rennie KL (2009) Added sugars and micronutrient dilution. Obes Rev 10, Suppl. 1, 34-40.

40. Frary CD, Johnson RK \& Wang MQ (2004) Children and adolescents' choices of foods and beverages high in added sugars are associated with intakes of key nutrients and food groups. J Adolesc Health 34, 56-63.

41. Alexy U, Kersting M \& Schultze-Pawlitschko V (2003) Two approaches to derive a proposal for added sugars intake for German children and adolescents. Public Health Nutr 6, 697-702.

42. Alexy U, Sichert-Hellert W \& Kersting M (2003) Associations between intake of added sugars and intakes of nutrients and food groups in the diets of German children and adolescents. Br J Nutr 90, 441-447.

43. Gibson SA (2007) Dietary sugars intake and micronutrient adequacy: a systematic review of the evidence. Nutr Res Rev 20, 121-131.

44. Bouhlal S, Issanchou S \& Nicklaus S (2011) The impact of salt, fat and sugar levels on toddler food intake. Br J Nutr $\mathbf{1 0 5}$, 645-653.

45. Sullivan SA \& Birch LL (1990) Pass the sugar, pass the salt: experience dictates preference. Dev Psychol 26, 546-551.

46. Foterek K, Hilbig A, Kersting M, et al. (2016) Age and time trends in the diet of young children: results of the DONALD study. Eur J Nutr 55, 611-620.

47. Hilbig A, Alexy U, Drossard C, et al. (2011) GRETA: Ernährung von Kleinkindern in Deutschland (German representative study of toddler alimentation). Aktuel Ernaehrungsmed 36, 224-231 (in German).

48. Smithers LG, Golley RK, Brazionis L, et al. (2011) Characterizing whole diets of young children from developed countries and the association between diet and health: a systematic review. Nutr Rev 69, 449-467.

49. Kranz S \& Siega-Riz AM (2002) Sociodemographic determinants of added sugar intake in preschoolers 2 to 5 years old. J Pediatr 140, 667-672.

50. Rasmussen LB, Matthiessen J, Biltoft-Jensen A, et al. (2007) Characteristics of misreporters of dietary intake and physical activity. Public Health Nutr 10, 230-237. 